\title{
Application of Neural Networks in Diagnosing Cancer Disease Using Demographic Data
}

\author{
Dr. N. Ganesan \\ Director (MBA/MCA), Regional \\ Institute of Cooperative \\ Management, Bangalore, India
}

\author{
Dr.K. Venkatesh \\ Head ,Department of Physics, M S \\ Ramaiah Institute of Technology, \\ Bangalore, India
}

A. Malathi Palani

Lecturer in Computer Science, M S

Ramaiah College of Art's Science and

Commerce, Bangalore, India.

\author{
Dr. M. A. Rama \\ Head, Department of Computer \\ Science, Maharani Lakshmi Ammani \\ College, Banqalore, India.
}

\begin{abstract}
Artificial Neural Network is a branch of Artificial intelligence, has been accepted as a new technology in computer science. Neural Networks are currently a 'hot' research area in medicine, particularly in the fields of radiology, urology, cardiology, oncology and etc. It has a huge application in many areas such as education, business; medical, engineering and manufacturing .Neural Network plays an important role in a decision support system. In this paper, an attempt has been made to make use of neural networks in the medical field (carcinogenesis (pre-clinical study)). In carcinogenesis, artificial neural networks have been successfully applied to the problems in both pre-clinical and post-clinical diagnosis. The main aim of research in medical diagnostics is to develop more cost-effective and easy-to-use systems, procedures and methods for supporting clinicians. It has been used to analyze demographic data from lung cancer patients with a view to developing diagnostic algorithms that might improve triage practices in the emergency department. For the lung cancer diagnosis problem, the concise rules extracted from the network achieve an high accuracy rate of on the training data set and on the test data set.
\end{abstract} Keywords:

Neural networks, fuzzy logic, carcinogenesis, lung cancer , rule extraction, back propagation ,medical decision making, decision support

systems.

\section{Introduction}

In recent years machine learning methods have been widely used in prediction, especially in medical diagnosis. Medical diagnosis is one of major problem in medical application. Several research groups are working world wide on the development of neural networks in medical diagnosis. Neural networks are used to increase the accuracy and objectivity of medical diagnosis. 'Neural networks' research and application have been studied for a half of hundred years

A detailed study on Artifical Neural Network (ANN) can be seen in "Neural and Adaptive Systems: Fundamentals Through Simulations "by Principe, Euliano, and Lefebvre(2000). Paulo J. Lisboa and Azzam F.G. Taktak (2006) had done a systematic review on artificial neural networks in decision support in cancer. This paper reports on a systematic review that was conducted to assess the benefit of artificial neural networks (ANNs) as decision making tools in the field of cancer. This paper reviews the clinical fields where neural network methods figure most prominently, the main algorithms featured, methodologies for model selection and the need for rigorous evaluation of results. Derong Liu; Zhongyu Pang; Lloyd, S.R (2008) developed an innovative signal classification method that is capable of differentiating subjects with sleep disorders which cause excessive daytime sleepiness (EDS) from normal control subjects who do not have a sleep disorder based on EEG and pupil size. Then, In addition to the use of Yoss pupil staging rule for scaling levels of wakefulness, an artificial neural network (NN) of modified adaptive resonance theory (ART2) is utilized to identify the two groups within a combined group of subjects including those with OSA and healthy controls. This results in $90 \%$ correct differentiation between narcoleptic and control subjects. Street W.N(2003), produced prediction results in to breast cancer data sets using a modification of ANN model. This model predicts probabilities of recurrence at different time intervals for each patient and has made an attempt to differentiate patients with 'good' or 'bad' prognosis. Sansanee Auephanwiriyakul ,Siripen Attrapadung,Sutasinee Thovutikul and Nipon Theera-Umpon (2005) trained Neural Network using back propagation and achieved an accuracy level on the test data of approximately $94 \%$ on breast cancer data. Seker .H., Odetao M.,Petroric D. and Naguib R.N.G(2003) presented another BP-ANN attempt where they used 47 input features and achieved an accuracy of 95\%. Zhi-Hua Zhou, Yuan Jiand (2003) have described a artificial network ensemble, which is helpful for utilizing the power of artificial neural network ensembles in reliable applications such as diabetes, hepatitis and breast cancer. In the review work of Kornel papik ,Zalan,Dombovari,Zsolt tulassay ,Janos feher and Bela molnar (1998), computer generated neural network and its applications have been described. A study the of applications of neural network in basic sciences, clinical medicine, signal processing and interpretation and medical image processing can be seen in Wan Hussain ,Wan Ishak (2002). Rusovick and Warner (1997) define telemedicine as any instance of medical care occurring via the internet and using real-time video-teleconferencing equipment as well as more specialized 
medical diagnostic equipment. Norsarini Salim (2004) discusses how Neural Network approach can diagnose disease using patient medical data such as breast cancer, heart failure, medical images, acidosis diseases, and lung cancer. Peter M. Ravdin, et al(1992) and Wu, Y., et al developed a neural network model to diagnose breast cancer. Frenster, J.H.(1990) described the Neural Network model for Pattern Recognition in Medical Diagnosis.

A method of selecting training data for neural network is presented in Hernandez, C.A. et al (1993) Eberhart, R. Micheli-Tzanako(1990) deals with various aspects and applications of neural networks in a wide spectrum of biomedical engineering problems. Meng Joo Er, Shiqian Wu , Juwei Lu\& Hock Lye Toh (1999) have developed efficient neural network approach using a radial basis function (RBF) neural classifier to cope with small training sets of high dimension. Jari J. Forsstrm , Kevin J. Dalton (1995) developed connectionist models such as neural networks, which define relationships among input data that are not apparent when using other approaches. They also reviewed the use of neural networks in medical decision support. Paulo J. Lisboa , Azzam F. G. Taktak(2006) assess the benefit of artificial neural networks (ANNs) as decision making tools in the field of cancer. In the work of G Wilym s. Lodwick,M.D.,Richard Conners and Charles A. Harlow (1979), an efficient neural network model has been developed to diagnosis the carcinogenesis. Neural network have been applied to breast cancer diagnosis. Kiyan and Yildirim(2003) employed Radial Basis Function, General Regression Neural Network and Probabilistic Neural Network in order to get the suitable result.

\section{Cancer disease pre-clinical Detection and Neural Networks}

Carcinogenesis (the creation of cancer), is the process by which normal cells are transformed into cancer cells. (In other words, uncontrolled and dangerous cell growth). Cancer is the general name for over 100 medical conditions involving uncontrolled and dangerous cell growth. One of the major determinants of an individual susceptibility to cancer is sex An obvious distinction that accounts for much of the variation in cancer incidence. There are a number of cancers to which only males are susceptible or to which only females are susceptible. For eg., females have no risk of ever experiencing cancer of prostrate, penis and males are not threatened by ovarian ,endometrial or cervical cancers. The next factor of susceptibility to cancer is age -Susceptibility to cancer is low for persons under thirty years of age and increases steadily in subsequent age groups, while middle aged persons and particularly older persons are more susceptible to cancer. The third factor is genetic predisposition - Some adult cancers show the effects of genetic transmission of susceptibility although other factors may be more prominently associated with their development. Lung cancer is an example of such inherited susceptibility. In case of female breast cancer, close relatives of breast cancer patients have a high risk of breast cancer two or three times that of women with no family history of breast cancer. The fourth factor considered is geographic variations - Scientists suggest that some cancer is caused by environmental conditions.
Today, cancer constitutes a major health problem. Lung cancer is one of the most common and deadly diseases in the world. It is the second leading cause of death .The most common risk factor for lung cancer is smoking, due to the harmful carcinogens found in tobacco smoke. Cancer of the lung is mostly related to smoking or tobacco use. When burned, cigarette smoke contains over 4000 chemicals, with over 60 of them being known carcinogens. Compared to nonsmokers, men who smoke are about 23 times more likely to develop lung cancer and women who smoke are about 13 times more likely. Smoking causes about $90 \%$ of lung cancer deaths in men and almost $80 \%$ in women. (For women, the risk of cervical cancer increases with the duration of smoking).

Neural networks are important tools for cancer detection and monitoring. The ability of the physicians to effectively treat and cure cancer is directly dependent on their ability to detect cancers at their earliest stages. An initial diagnosis called early diagnosis is made based on the demographic and clinical data of the patient. More than $30 \%$ cancer deaths are preventable. Curing cancer has been a major goal of medical researchers for decades, but development of new treatment takes time and money. Science may yet find the root causes of all cancers and develop safer methods for shutting them down before they have a chance to grow or spread. Artificial neural networks offer a completely different approach to problem solving and they are sometimes called the sixth generation of computing. The aim of this research is to apply neural networks and their associated analysis techniques to Health care, specifically to the management of lung cancer patients. Artificial neural networks now are used in many fields. They have become well established as viable, multipurpose, robust computational methodologies with solid theoretic support and with strong potential to be effective in any discipline, especially medicine. Over the last two decades, a tremendous amount of research work has been conducted for automated cancer diagnosis. Chiou et al.(2003) designed an artificial neural network based hybrid lung cancer detection system named HLND, which improves the accuracy of diagnosis and the speed of lung cancerous pulmonary radiology. It includes the processing phases, such as pre-processing to enhance the figurebackground contrast, quick selection of nodule suspects based upon the most prominent feature of nodules and complete feature space determination and neural classification of nodules. Gutte, Henrik (2007) developed a completely automated method based on image processing techniques and artificial neural networks for the interpretation of combined fluorodeoxyglucose (FDG) positron emission tomography (PET) and computed tomography (CT) images for the diagnosis and staging of lung cancer. Kenji Suzuki(2003) investigated a pattern-recognition technique based on an artificial neural network (ANN). Penedo et al (1998) developed a system that employed an artificial neural network to detect suspicious regions in a low-resolution image and employed another artificial neural network to deal with the curvature peaks of the suspicious regions, which was used in the detection of lung nodules found on digitized chest radiographs. Bartfay (2006) proposed a neural network model. Utilizing data on patients from two National Cancer Institute of Canada clinical trials, he compared predictive accuracy of neural network models and logistic regression models on risk of death of limited-stage small-cell lung cancer patients. 


\section{Background: Neural Network}

The construction of the neural network involves three different layers with feed forward architecture. This is the most popular network architecture in use today. The input layer of this network is a set of input units, which accept the elements of input feature vectors. The input units(neurons) are fully connected to the hidden layer with the hidden units. The hidden units (neurons) are also fully connected to the output layer. The output layer supplies the response of neural network to the activation pattern applied to the input layer. The information given to a neural net is propagated layer-by-layer from input layer to output layer through (none) one or more hidden layers.

Important issues in MultiLayer Perceptrons (MLP) design include specifications of the number of hidden layers and the number of units in these layers. The number of input and output units is defined by the problem the number of hidden units of use is far from clear. That is the amount of hidden layers and their neurons is more difficult to determine. A network with one hidden layer is sufficient to solve most tasks. The universal approximation theorem for neural networks states that every continuous function that maps intervals of real numbers to some output interval of real numbers can be approximated arbitrarily closely by a multi-layer perception with just one hidden layer. There is no theoretical reason ever to use more than two hidden layers. It is also been seen that for the vast majority of principal problems . Those problems that require two hidden layers are only rarely encountered in real life situations. Using more than one hidden layer is almost never beneficial. It often slows dramatically when more hidden layers are used .None of the known problems needs a network with more than three hidden layers in order to be solved error. Choosing an appropriate number of hidden nodes is important. A common approach is to start with a larger number of nodes and then employ network pruning algorithms for optimization. An excessive number of hidden neurons may cause a problem called 'overfitting'. L Rawtani (2001) suggested choosing the number of hidden nodes should be one or more than the training points on the curve. The number of neurons in the hidden layers may equivalent to the control points. Here the average number of control points (4) has been assigned as the number of hidden neurons.

\section{The Model}

Artificial Neural Network model could perform "intelligent" tasks similar to those performed by the human brain. Artificial neural network models offer a completely different approach to problem solving and they are sometimes called the sixth generation of computing. In the network the input neuron values are the demographic data concerns information such as patient's age, sex etc. The hidden neuron values are based on heuristic diagnostic knowledge represents experience accumulated through years and concerns the way an expert uses the patient data to make diagnoses. The heuristic knowledge has been acquired interviewing experts in the field and constructed a diagnostic tree based on criteria. A feature vector $\mathrm{x}$ consists of $\mathrm{n}$ components denoted by $\mathrm{x}_{1}$, $\mathrm{x}_{2}, \mathrm{x}_{3} \ldots$ where $\mathrm{x}_{\mathrm{i}}$ represents a variable that is relevant to the classification problem. Nominal variables are used to represent the input values in the nodes of the input layer. Nominal variables may be two-state or many-state. A twostate nominal variable is easily represented by transformation into a numeric value. In this number of numeric variables represent the single nominal variable. The number of numeric variables equals the number of possible values; one of the $\mathrm{N}$ variables is set and the others are cleared. Neural networks has facilities to convert both two-state and many-state nominal variables. They can be represented using an encoding known as one-of-N encoding. The hidden layer contains four hidden neurons $h_{1}, h_{2}, h_{3}$ and $h_{4}$ which represent the symptoms of lung cancer. The output layer contains two neurons that produce the binary outputs.

\section{Training the model}

Once a network has been structured for a particular application, that network is ready to be trained. To start this process the initial weights are chosen randomly. Then, the training, or learning, begins. The ANN has been trained by exposing it to sets of existing data (based on the follow up history of cancer patients) where the outcome is known. Multi-layer networks use a variety of learning techniques; the most popular is back - propagation algorithm. It is one of the most effective approaches to machine learning algorithm developed by David Rummelhart and Robert McLelland (1994). Information flows from the direction of the input layer towards the output layer. A network is trained rather than programmed. Learning in ANN's is typically accomplished using examples. This is also called 'training' in ANN's because the learning is achieved by adjusting the connection weights in ANN's iteratively so that trained (or learned) .The number of iterations of the training algorithm and the convergence time will vary depending on the weight initialization. After repeating this process for a sufficiently large number of training cycles the network will usually converge to some state where the error of the calculations is small. In this case one says that the network has learned a certain target function. Learning techniques are often divided into supervised, unsupervised and reinforcement learning. 
The neural network structure is shown in the following figure:

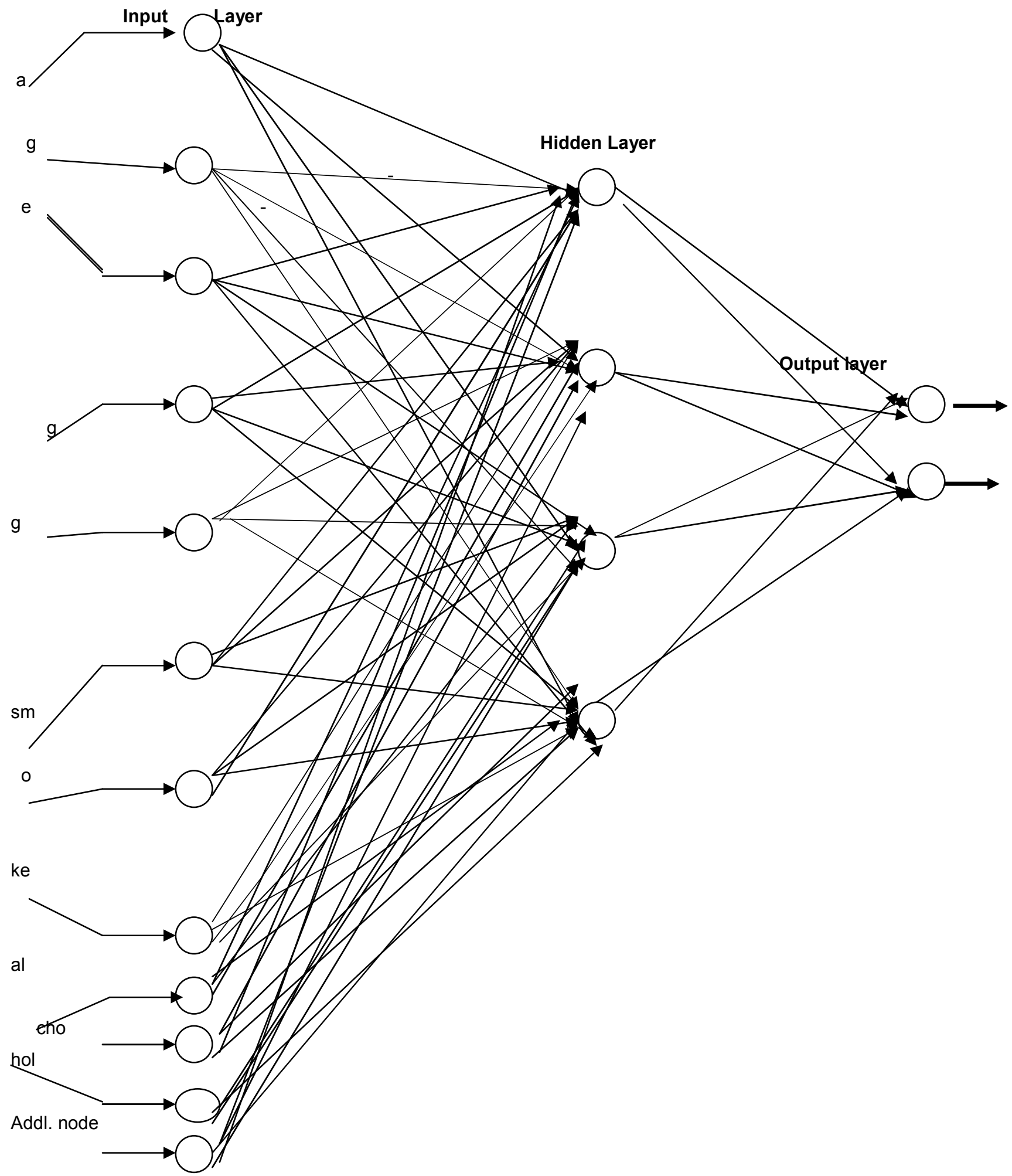


Nominal variables are used to represent the input values in the the nodes of the input layer. Nominal variables may be two-state or many-state.A two-state nominal variables is easily represented by transformation into a numeric value .For e.g, Male $=0$, Female $=1$.Many-state nominal variables are more difficult to handle.Thay can be represented using an encoding .known as one-of-N encoding In this number of numeric variables represent the single nominal variable.The number of numeric variables equals the number of possible values ; one of the $\mathrm{N}$ variables is set and the others are cleared. For e.g age $<35=\{1,0,0\}$, age $>=35<=55$ $=\{0,1,0\}$, age $>55=\{0,0,1\}$. Similarly the other input values are represented .Neural networks has facilities to convert both two-state and many-state nominal variables .

It is well known that the performance of learning systems on the training data often does not reflect the performance on unknown data. This is due to the fact that the system often adapts well on training to the particularities of the training data. Therefore, the training data should be randomly chosen from all available data. It should represent the typical data properties. If we have initially a bias in the training data you will encounter performance problems for the test data later. In order to test the real generalization abilities of a network to unknown data, it must be tested by classified, but yet unknown data, the test data that should not contain samples coming from patients of the training data. We have to face the fact that patient data is very individual and it is difficult to generalize from one patient to another. Ignoring this fact would pretend better results than a real system could practically achieve.

\section{Selection of weights}

Initially, the weights on all the interconnections are set to be small random numbers [Carlos Hernandez 2001], and the network is said to be "untrained." The weights of each neuron (node) were randomly initialized to values between 1 and +1 . The last layer is the output layer, and activation levels of the neurons in this layer are considered to be the output of the neural network. The network then is presented with a training data set, which provides inputs and desired outputs to the network. Weight training in ANN's is usually formulated as minimization of an error function [xin] has the mean square error between target and actual outputs averaged overall examples, by iteratively adjusting connection weights. Weights are adjusted in such a way that each weight adjustment increases the likelihood that the network will compute. To adjust weights is calculated and the weights are then changed such that the error decreased (thus going downhill on the surface of the error function). The probability of successful convergence will depend on the weight initialization scheme. For this reason backpropagation can only be applied on networks with differentiable activation functions.

$$
\begin{array}{r}
\mathrm{S}_{\mathrm{j}}=\sum_{i=0}^{n} w_{j i a} a_{i} \\
a_{j}=\frac{1}{1+\exp \left(-S_{j}\right)}
\end{array}
$$

Typically, initial small random weights are updated gradually.

A small change is done at each cycle of the network training phase, which is guided by the direction (signal) of the error, and a constant of proportionality(or learning rate)according to where $\Delta$ is the difference between target (desired output) and real output, and a is the value entered in the input unit. The updated weight is calculated by summing the w and the initial weight. The basic idea underlying the learning algorithms usually utilized in neural networks is simple. The proposed model is trained with back propagation algorithm. The error backpropagation (EBP) training of a multilayer perceptron (MLP) may require a very large number of training epochs. Although the training time can usually be reduced considerably by adopting an on-line training paradigm, it can still be excessive when large networks have to be trained on lots of data, it offers improved accuracy, speed, and robustness against badly scaled inputs. A major characteristic of EBP is its utilization of weight specific learning rates whose relative magnitudes are derived from a priori computable properties of the network and the training data Martens, J.-P. Weymaere, N (2002). The model starts with small random real numbers as the starting weights. At each training cycle, the error is calculated, and the weights are changed in the direction that minimizes the error. The error surface has as many dimensions as the number of weights, and all the weights obey this basic principle.This process of changing the weights or updating the weights is called training.All weights are kept until the end of training.Training is an external process.Learning is the internal process.Learning in neural networks is performed by iteratively modifying weights such that the desired output is eventually produced by the network, with a minimal amount of error.(John Paul 2006) It is almost never necessary to continue training until the training error stops decreasing; the best performance on the development set will almost always occur at an earlier iteration. Also best performance on the development set occurs after about 20 to 30 iterations, and so training is done for a fixed number of iterations, usually between 30 and 45 .

\section{Data description and Training data}

MATLAB is derived from MATrix LABoratory. The MatLab programming language is exceptionally straightforward since almost every data object is assumed to be an array It is an interactive, matrix-based system for scientific and engineering numeric computation and visualization. The data obtained from various hospitals were used for this study. Initially fifty samples are given in matlab. Matlab relieves you of a lot of the mundane tasks associated with solving problems numerically. Powerful operations can be performed using just one or two 
commands. . It includes high-level functions for twodimensional and three-dimensional data visualization, image processing, animation, and presentation graphics.

Initially 100 lung cancer patients data has been collected from various hospitals and trained with the neural networks.
It gives more than $87 \%$ of accuracy. The present algorithm is fast ,taking only few seconds of execution time . The results are found to be better using back propagation algorithm. In this paper, an attempt has been made to make use of neural networks in the medical field
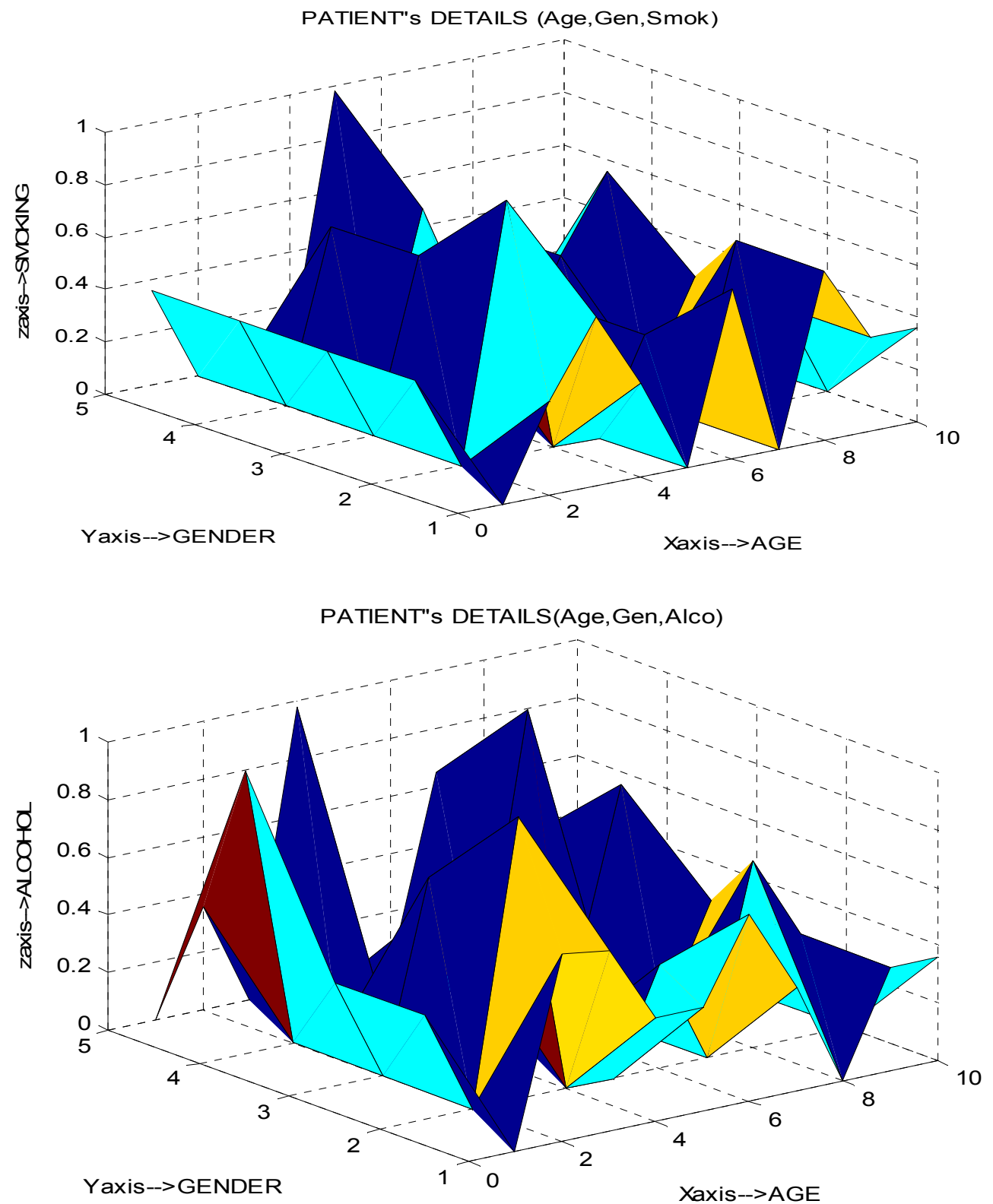

\section{CONCLUSION}

Lung cancer is one of the most common and deadly diseases in the world.Detection of lung cancer in its early stage is the key of its cure. The automatic diagnosis of lung cancer is an important, real-world medical problem.In this paper the author has shown how neural networks are used in actual clinical diagnosis of lung cancer .Neural network model, a diagnostic system that performs at an accuracy level is constructed here. In this work, the performance of neural network structure was investigated for lung cancer diagnosis problem. People can be checked for lung cancer disease quickly and painlessly and thus detecting the disease at an early stage. Of course, They would become perfect 'doctors in a box', assisting or surpassing clinicians with tasks like diagnosis. This work indicates that neural network can be effectively used for lung cancer diagnosis to help oncologists. The prediction could help doctor to plan for a better medication and provide the patient with early diagnosis. 


\section{REFERENCES}

1. Anagnostou T, Remzi M, Lykourinas M, Djavan B (2003). "Artificial neural networks for decision-making in urologic oncology”. Eur Urol. 2003 Jun;43(6):596-603. Review.

2. Antognetti, P. and Milutinovic, V.(1997), "Neural Networks: Concepts, Applications, and Implementations (Eds.)" Volumes I-IV, Prentice Hall, Englewood Cliffs, NJ., 1991.

3. A. Tewari. Artificial intelligence and neural networks: Concept, applications and future in urology. British Journal of Urology, 80 Supplement 3:53-8, 1997.

4. Baker J A, Kornguth PJ, Lo JY, Williford ME, Floyd $\boldsymbol{C E} \boldsymbol{J r}(1995)$ : "Breast cancer: prediction with artificial neural network based on BI-RADS standardized lexicon". Radiology, 1995; 196(3): 817-22

5. Bartfay, E. Ph.D; Mackillop, W. J. MBCHB; Pater, J. L(2006). MDMSC- "Comparing the predictive value of neural network models to logistic regression models on the risk of death for small-cell lung cancer patients" ,ASSOCIATE PROFESSOR, University of Ontario Institute of Technology, Oshawa, Ontario, Canada (BARTFAY),DIVISION DIRECTOR, Queen's Cancer Research Institute, and professor and head, Department of Community Health \& Epidemiology, Queen's University, Kingston, Ontario, Canada (MACKILLOP) (PATER)-: Emma Bartfay, Faculty of Health Sciences, University of Ontario Institute of Technology, Oshawa, ON L1H 7K4, Canada (e-mail: emma.bartfay@uoit.ca),,European Journal of Cancer Care:Volume 15(2)May 2006p 6. B. Cheng and D.M. Titterington (1994). Neural networks:" A review from a statistical perspective". Statistical Science, 9:2-54, 1994.

7. B.D. Ripley (1996). "Pattern Recognition and Neural Networks". Cambridge University Press, Cambridge, 1996.

8. B.D. Ripley (1993). "Statistical aspects of neural networks". In O.E. Barndorff-Nielsen, J.L.Jensen, and W.S. Kendell, editors, Networks and Chaos - Statistical and Probabilistic Aspects, pages 40-123. Chapman \& Hall, London, 1993.

9. B.D. Ripley and R.M. Ripley(2001) . "Neural networks as statistical methods in survival analysis". In R. Dybowski and V. Gant, editors, Clinical Applications of Artificial Neural Networks, chapter 9. Cambridge University Press, Cambridge. In press.

10. Brown, Robert J (1987)., "An Artificial Neural Network Experiment", Dr. Dobbs Journal, April 1987.52. Rawtani,J LRana \&A K Tiwari-Number Hidden Nodes for Shape Preserving ANN Representation of a Curve,Maulana Azad College of Technology ,Dept. of Electronics and Computer Science \& Engineering,Bhopal,India

11.Carlos Hernandez-Espinosa,Mercedes FernandezRedondo (2001) -Universidad Jaume I.Campus de Riu Sec,Spain,IEEE 2001

12. C.A. Galletly, C.R. Clark, and A.C. McFarlane (1996). Artificial neural networks: "A prospective tool for the analysis of psychiatric disorders". Journal of Psychiatry \& Neuroscience, 21(4):239-47, 1996.

13. Chiou YSP, Lure YMF (1993), Ligomenides PA. "Neural network image analysis and classification in hybrid lung nodule detection (HLND) system". In: Proceedings of the IEEE-SP Workshop on Neural Networks for Signal Processing, 1993.

14. C.M. Bishop, M. Svensen, and C.K.I. Williams (1997). GTM: "The generative topographic mapping". Neural Computation, 10(1):215-234, 1997.

15. C.Pappas, N.Maglaveras and J.R.Scherrer(1998), "The computational capabilities of three-layered neural networks"- IOS press, Thessalonike, Greece.were proven by Hornik et al.,10

16. C. Robert, C. Guilpin, and A. Limoge (1998) . "Review of neural network applications in sleep research". Journal of Neuroscience Methods, 79(2):187-193, 1998.

17. Derong Liu; Zhongyu Pang; Lloyd S.R (2008) -“A Neural Network Method for Detection of Obstructive Sleep Apnea and Narcolepsy"- Based on Pupil Sizeand EEG.2008 V-19 I-2

18. D. Itchhaporia, P.B. Snow, R.J. Almassy, and W.J. Oetgen(1996) . "Artificial neural networks: Current status in cardiovascular medicine". Journal of the American College of Cardiology, 28(2):515-521, 1996.

19. D.J.C. MacKay (1992). "A practical Bayesian framework for back-propagation networks. Neural Computation", 4(3):448-472, 1992.

20. Djoussé L et al.(2002) Alcohol Consumption and Risk of Lung Cancer: "The Framingham Study". J Natl Cancer Inst 2002;94:1877-82.

21. Dreifus $\boldsymbol{L S}$ (1956) . "A clinical correlative study of the electrocardiogram in electrolyte imbalance". Circulation.1956; 14: 815-825.

22. E. BARTFAY, PHD, ASSOCIATE PROFESSOR ${ }^{1}$, W.J. MACKILLOP, MB , CHB , DIVISION DIRECTOR ${ }^{2} \&$ J.L. PATER, MD , MSC , DIVISION DIRECTOR ${ }^{3}(\mathbf{2 0 0 6})$ "Comparing the predictive value of neural network models to logistic regression models on the risk of death for smallcell lung cancer patients", ' University of Ontario Institute of Technology, Oshawa, Ontario, ${ }^{2}$ Queen's Cancer Research Institute, and professor and head, Department of Community Health \&Epidemiology,Queen'sUniversity,Kingston,Ontario,

Queen's Cancer Research Institute, Department of Oncology, Queen's University, Kingston, Ontario, Canada .

23. Eberhart, $R$. Micheli-Tzanako, E (1990).-"Neural networks for engineering in medicine and biology ",Appl. Phys. Lab., Johns Hopkins Univ., Laurel, MDIEEETransactionson Volume 1, Issue 4,Dec1990Page(s):305 - 306 24. Martens, J.-P. Weymaere, $N$ (2002)-“An equalized error backpropagation algorithm for the on-line training of multilayer perceptrons".Electronics \& Inf. Syst., Ghent Univ., Gent; IEEE trans, 2002.

25. Fausett, L (1994). "Fundamentals of Neural Network: Architectures, Algorithms and Applications". Prentice Hall; Englewood Cliffs(1994).

26. Flexer.A (1995) , "Connectionists and statisticians", friends or foes In J. Mira and F. Sandoval, editors, Proceedings of the International Workshop on Artificial Neural Networks, LNCS 930, pages 454-461. Springer, 1995.

27. Fogel DB, Wasson EC (1995) 3rd, Boughton EM:

"Evolving neural networks for detecting breast cancer".

Cancer Lett, 1995; 96(1): 49-53

28. Frenster, J.H (1990)., "Neural Networks for Pattern 
Recognition in Medical Diagnosis", Annual International Conference in the IEEE Engineering in Medicine and Biology Society, vol. 12, No. 3, issued 1990, pp. 1423-1424 29. Greenlee RT, Murray T, Bolden S(2000), et al.: Cancer statistics 2000. CA Cancer J Clin 2000, 50:7-33

30. Gutte, Henrik ${ }^{a}$; Jakobsson, David ${ }^{b}$; Olofsson, Fredrik ${ }^{b}$; Ohlsson, Mattias ${ }^{b}{ }^{c}$; Valind, Sven ${ }^{b}$ Loft, Annika ${ }^{a}$; Edenbrandt, Lars ${ }^{b d}$; Kjaer, Andreas ${ }^{a}$ (2007)' - "Automated interpretation of PET/CT images in patients with lung cancer"- Departments of 'Clinical Physiology, Nuclear Medicine and PET, Rigshospitalet, Copenhagen University Hospital, Denmark ${ }^{b}$ Clinical Physiology, Malmö University Hospital, Sweden ${ }^{\mathrm{c}}$ Theoretical Physics, Lund University, Sweden ${ }^{\mathrm{d} C l i n i c a l}$ Physiology, Sahlgrenska University Hospital, Göteborg, Sweden ${ }^{\mathrm{e} C l u s t e r}$ for Molecular Imaging, University of Copenhagen, Denmark, Correspondence to Dr Henrik Gutte, Department of Clinical Physiology, Nuclear Medicine and PET, Rigshospitalet, Copenhagen University Hospital, Denmark-Nuclear Medicine Communications:Volume 28(2)February 2007.

31. G Wilym s(1). Lodwick,M.D.,Richard Conners(2) ,Ph.D and Charles A. Harlow(2),Ph.D-(!) Dept. of Radiology ,Univ. of Missouri, Colunbia (@)Dep of Electrical Engg. ,Louisiana state univ.,Louisiana -IEEE 1979

32. Hatzilygerodudis, P.J.Vassilakos, A.tsakalidis (1997)XBONE: "A Hybrid Expert System Supporting Diagnosis of Bone Diseases"- University of patras, school of engineering, dept of computer engine.\& informatics, Greece and computer technology institute, Greece. Regional university hospital of patras, dept of nuclear medicine patras Greece.Published in the proceedings of the medical informatics europe'97

33. Heine H. Hansen, MD(1990)- Lung Cancer-“A Changing Picture",National University Hospital, The Finsen Center 5072,9 Blegdamsvej, Copenhagen DK-2100, 1990 ,Denmark.E-mail: hansenhh@iaslc.org

34. Hernandez, C.A. et al (1993)., "How to Choose the Training Data for Neural Network Medical Diagnosis Systems", ISA, pp. 283-290 (1993)

35. Hornik K, Stinchcomb X(1989), White X. "Multilayer feedforward networks are universal approximators". Neural Net 1989;2: 359-66.

36. Ismail SARITAS, Novruz ALLAHVERDI and Ibrahim Unai SERT(2003) - "A Fuzzy System Design for diagnosis of Prostrate Cancer",International Conference on Computer systems and Technologies-CompSysTech'2003

37. Jan Baumbach1,2, Alexander Bunkowski1, Sita Lange1,3, Timm Oberwahrenbrock1, Nils Kleinb * olting1,3, Sven Rahmann1, J “ org Ingo Baumbach4 IMS2 (2007)- "An integrated medical software system for early lung cancer detection using ion mobility spectrometry data of human breath "1 Computational Methods for Emerging Technologies, Genome Informatics, Technische Fakult " at, Bielefeld University, 33594 Bielefeld, Germany 2 International NRW Graduate School in Bioinformatics and Genome Research, Bielefeld University 3 Bioinformatics Resource Facility, Bielefeld University

4 Department of Metabolomics, ISAS - Institute for Analytical Sciences,

Bunsen-Kirchhoff-Str. 11, 44139 Dortmund, Germany Journal of Integrative Bioinformatics 2007

38. Jari J. Forsstrm ${ }^{a}$; Kevin J. Dalton ${ }^{b}$ (1995) -“Artificial
Neural Networks for Decision Support in Clinical Medicine" - Department of Medicine, University of Turku, Finland-(a)Obstetrics and Gynaecology, Division of Materno-Fetal Medicine, (b)University of Cambridge, Rosie Maternity Hospital, Cambridge, UK,Annals of Medicine Vol 27,I 5,1995.

39. Javed Khan1,2, Jun S. Wei1, Markus Ringner1,3, Lao H. Saal1, Marc Ladani4, Frankwestermann 5, Frank Berthold 6, Manfred Schwab 5, Cristina R. Antoescu 4, Carsten Peterson 3 \& Paul S. Meltzer 1 (2001)Classification and diagnostic prediction of cancers using gene expression profiling and artificial neural networks ,1Cancer Genetics Branch, National Human Genome Research Institute,National Institutes of Health, Bethesda, Maryland, USA 2Pediatric Oncology Branch, Advanced Technology Center, National Cancer Institute, Gaithersburg, Maryland, USA-2001 ,3Complex Systems Division, Department of Theoretical Physics, Lund University, Lund, Sweden 4Department of Pathology, Memorial SloanKettering Cancer Center, New York, New York, USA 5Department of Cytogenetics, German Cancer Research Center, Heidelberg, Germany,6Department of Pediatrics, Klinik für Kinderheilkunde der Universität zu Köln, Köln, Germany

40. J.L.Jensen, and W.S. Kendell (1993), editors, Networks and Chaos - Statistical and Probabilistic Aspects, pages 139. Chapman \& Hall, London, 1993. 41.John-Paul Hosom, Jacques de Villiers, Ron Cole, Mark Fanty, Johan Schalkwyk, Yonghong Yan, Wei Wei(2006),' Training Hidden Markov Model/Artificial Neural Network (HMM/ANN) Hybrids for Automatic Speech Recognition "(ASR)-Center for Spoken Language Understanding (CSLU) .OGI School of Science \& Engineering (OGI)Oregon Health \& Science University (OHSU) (feb 2006)

41. Jose C. Principe,Neil R. Euliano and W. Curt Lefebvre(2000)-"Neural and Adaptive Systems: Fundamentals Through Simulations",published by John Wiley and Son(2000).

42. J.P. Hogge, D.S. Artz, and M.T. Freedman(1997). "Update in digital mammography.Critical Reviews in Diagnostic Imaging”, 38(1):89-113, 1997.

43. J.S. Bridle(1991). "Probabilistic interpretation of feedforward classification network outputs, with relationships to statistical pattern recognition". In F. Fogleman- Soulie and J. Herault, editors, Neurocomputing: Algorithms, Architectures, and Applications, pages 227236. Springer-Verlag, Berlin, 1991.

44. J.T. Wei, Z. Zhang, S.D. Barnhill, K.R. Madyastha, H. Zhang, and J.E. Oesterling(1998). "Understanding artificial neural networks and exploring their potential applications for the practicing urologist”. Urology, 52(2):161-172, 1998.

45. J. Wyatt. Nervous(2001) "About artificial neural networks"? The Lancet ,2001.

46. Kenji Suzuki, Samuel G. Armato III, Feng Li, Shusuke Sone, and Kunio Doi (2003) -"Massive training artificial neural network (MTANN) for reduction of false positives in computerized detection of lung nodules in low-dose computed tomography"- Kurt Rossmann Laboratories for Radiologic Image Research, Department of Radiology, The University of Chicago, Chicago, Illinois 60637-Medical Physics, Volume 30, Issue 7, (July 2003) 
47. Kiyan, T., and Yildirim, T. (2003). Breast Cancer Diagnosis Using Statistical Neural Networks. International XII. Turkish Symposium on Artificial Intelligence and Neural Networks. University Besiktas, Istanbul, Turkey.2003.

48. Kornel papik, Zalan,Dombovari,Zsolt tulassay, ,Janos feher and Bela molnar(1998) - Dept of medicine,Semmaleveis medical university,Hungary and Raier Schaefer- Germany - "Applications of neural networks in medicine"-a review 1998-Vol 4.

49. L. Aakerlund and R. Hemmingsen(1998)." Neural networks as models of sychopathology" .Biological Psychiatry, 43(7):471-82, 1998.49. L Rawtani,J LRana \& A $\boldsymbol{K}$ Tiwari(2001) -"Number Hidden Nodes for Shape Preserving ANN Representation of a Curve",Maulana Azad College of Technology ,Dept. of Electronics and Computer Science \& Engineering,Bhopal,India,2001.

50. L. Tarassenko.(1998)- "A Guide to Neural Computing Applications". Arnold, London, 1998.

51. L. Tarassenko(1995). "Neural networks". Lancet, 346:1712, 1995.

52. L. Tarassenko, P. Hayton, N. Cerneaz, and M. Brady(1995). "Novelty detection for the identification of masses in mammograms". In Proceedings of the 4th IEE International Conference on Artificial Neural Networks, pages 442-447, Cambridge,1995. Cambrdige Univeristy Press.

53. Meng Joo Er, Shiqian Wu, Juwei Lu\& Hock Lye Toh(1999)- "Face recognition with radial basis function (RBF) neural networks". School of Electricals \& Electronics Engineering, Nanyang Technol. Univ., Singapore Decision and Control, 1999. Proceedings of the 38th IEEE Conferenceon Volume 3, Issue , 1999 Page(s):2162 - 2167

54. MF Wu, JJ Chiang1,2, YC Yang, IH Chao, SM Shieh2, WC Tzeng, Hsieh(2001)- "Predicting Hyperkalemia by a Two-Staged Artificial Neural Network"1Department of Bioinformatics, Chung Hua University, Hsin-Chu, Taiwan,2Wei Gong Memorial Hospital, Miao-Li, Taiwan 45. Poters RS etc. Prediction of hyperkalemia in dogs from electrocardiographic parameters using an artifificial neural network. Academic Emergency Medicine. 2001; 8(6):599-603.

55. Moul JW, Snow PB, Fernandez EB, Maher PD, Sesterhenn IA(1995)- "Neural network analysis of quantitative histological factors to predict pathological stage in clinical stage I nonseminomatous testicular cancer". J Urol, 1995; 153(5): 1674-5 Philip

56. M.R. Brickley, J.P. Shepherd, and R.A. Armstrong(1998). Neural networks: "A new technique for development of decision support systems in dentistry". Journal of Dentistry, 26(4):305-309, 1998.

57. S.-I. Amari(1991). "Mathematical methods of neurocomputing". In O.E. Barndorff- Nielsen, 53. S. Maclin, Jack Dempsey, Jay Brooks, and John Rand (1991)-“Using Neural Networks to Diagnose Cancer"-While artificial brains are in the realm of science fiction, artificial neural networks (ANNs) are scientific facts. An artificial neural Journal of Medical Systems, Vol. 15, No. 1, 1991

58. N.A. Khan, F. Afroz, M.M. Lone, M.A. Teli, M. Muzaffar and N. Jan(2006) -"study by Department of Radiation Oncology", Sher-i-Kashmir Institute of Medical Sciences, Srinagar, India-2006
59. Nauck/KrusE(1998)-"How the Learning of Rule Weights Affects the Interpretability of Fuzzy Systems"Paper appears in Proc. IEEE International Conference on Fuzzy Systems 1998 (FUZZ-IEEE'98), Anchorage, AK, May 4-9, 1998.

60. Norsarini Salim(2004) - "Medical Diagnosis Using Neual Networks "-Faculty of Information Technology,University of Utara Malaysia(2004).

61. Paulo J. Lisboa , Azzam F. G. Taktak(2006)-“The use of artificial neural networks in decision support in cancer"A systematic review- - School of Computing and Mathematical Science, Liverpool John Moores University, Liverpool, UK, Department of Clinical Engineering, Royal Liverpool University Hospital, Liverpool, UK ,Neural Networks-Volume 19 Issue 4 (May 2006) ---Penedo MG, Carreira MJ, Mosquera A, Cabello D. Computer-aided diagnosis: a neural-network-based approach to lung nodule detection. IEEE Trans. Medical Imaging 1998; 17(6)

62. Penedo MG, Carreira MJ, Mosquera A, Cabello D(1998)." Computer-aided diagnosis: a neural-networkbased approach to lung nodule detection". IEEE Trans. Medical Imaging 1998; 17(6)

63. Penny W etc(1996)." Neural networks in clinical medicine". Med Decis Making. 1996; 16:386-398.

64. Peter M. Ravdin, et al(1992), "A practical application of neural network analysis for predicting outcome of individual breast cancer patients", Breast Cancer Research \& Treatment, vol. 22, pp. 285-293 (1992)

65. P.H. Goodman and F.E. Harrell.(1998)- Neural networks-"Advantages and limitations for biostatistical modeling". Technical report, Washoe Medical Center, University of Nevada, October 1998.

66. P.Y. Ktonas(1996). "Computer-based recognition of EEG patterns". -Electroencephalography \& Clinical Neurophysiology - Supplement, 45:23-35, 1996.

67. Ravdin PM, Clark GM, Hilsenbeck SG et al(1992)- "A demonstration that breast cancer recurrence can be predicted by neural network analysis”. Breast Cancer Res Treat, 1992; 21(1): 47-53

68. R. Dybowski and V. Gant(1995). "Artificial neural networks in pathology and medical laboratories". Lancet, 346:1203-1207, 1995.

69. R. Goodacre, M.J. Neal, and D.B. Kell(1996). "Quantitative analysis of multivariate data using artificial neural networks": A tutorial review and applications to the deconvolution of pyrolysis mass spectra. Zentralblatt fur Bakteriologie,284(4):516-539, 1996.

Ries LAG, Melbert D, Krapcho M, Stinchcomb DG, Howlader N, Horner MJ, Mariotto A, Miller BA, Feuer EJ, Altekruse SF, Lewis DR, Clegg L, Eisner MP, Reichman M, Edwards BK (eds). SEER Cancer Statistics Review, 19752005, National Cancer Institute. Bethesda, MD, based on November 2007 SEER data submission, posted to the SEER Web site, 2008.

R.N. Naguib and G.V. Sherbet. Artificial neural networks in cancer research. Pathobiology, 65(3):129-139, 1997.

70. Rusovick, R., and Warner, D. (1997). The Webification of Medicine: Interventional InformaticsThroughtheWWW.http://www.pulsar.org/febwe b/papers/mwww3.htm

$\begin{array}{lcr}71 . \quad \text { Sansanee } & \text { Auephanwiriyakul } & \text { Siripen } \\ \text { Attrapadung,Sutasinee } & \text { Thovutikul(2005) } & \text {-Computer }\end{array}$


engineering department, and Nipon Theera-Umpon Electrical engineering department Chiang Mai University, Thailand- "Breast abnormality detection in mammograms using fuzzy inference system",2005.

72. Seker .H., Odetao M.,Petroric D. and Naguib R.N.G.(1994)- "A fuzzy logic based method for prognostic decision making in breast and prostrate cancers"Biomedicine (IEEE transactions) 2003.73. S. Haykin. Neural Networks: A Comprehensive Foundation. Macmillan, New York, 1994.

74. Street $\boldsymbol{W} \cdot \boldsymbol{N}(\mathbf{1 9 9 8 )}$ - "Artificial Neural Network model for prognostic prediction" ,Proceedings of $15^{\text {th }}$ International conference on ML -Fuzzy logic based method for prognostic decision making in breast and prostrate,1998. cancersBiomedicine (IEEE transactions) 2003.

75. U.S Department and Human services (2004). The health consequences of smoking:"A report of the surgeon general". U S Department of health and human services ,centers for disease control and prevention ,National center for chronic disease prevention and health promotion, office on smoking and health,2004.

76. Wan Hussain ,Wan Ishak(2002)-“The Potential of Neural Networks in Medical Applications" -Faculty of Information Technology,Universiti Utara Malaysia, 06010 Sintok, Kedah, MALAYSIA(2002).
77. W. el Deredy(1997) -"Pattern recognition approaches in biomedical and clinical magnetic resonance spectroscopy": A review. NMR in Biomedicine, 10(3):99-124, 1997.

78. W. Penny and D. Frost(1996) - "Neural networks in clinical medicine". Medical Decision Making, 16(4):386389, 1996.

79. Wrenn KD etc(1991). "The ability of physicians to predict hyperkalemicnfrom the ECG”. Ann Emerg Med. 1991;20:1229-1232.

80. $\boldsymbol{W} \boldsymbol{u}, \boldsymbol{Y}$., et al.(1993), Artificial Neural Networks in Mammorgraphy: "Application to Decision Making in the Diagnosis of Breast Cancer", Radiology, vol. 187, pp. 81-87 1993)

81. W.W. Tryon.(1998) - “A neural network explanation of posttraumatic stress." Journal of Anxiety Disorders, 12(4):373-85, 1998.

82. Xin Yao (1999)_“Evolving Artificial Neural Networks"-Senior memberProceedings of the IEEE,Sep 1999,Vol-87,IEEE

83. Yang TF etc(1994). "Use of artificial neural networks within deterministic logic for the computer Eferior myocardial infarction". Journal of Electrocardiology 1994; 27:188-193.

84. Zhi-Hua Zhou, Yuan Jiand (2003)- "Medical diagnosis with C4.5 rule preceded by ANN ensemble", (IEEE)Information technology in biomedicine,2003. 